\title{
Metrology for ammonia in ambient air - concept and first results of the EMRP project MetNH3
}

\author{
Andrea Pogány ${ }^{1}$, David Balslev-Harder ${ }^{2}$, Christine F. Braban ${ }^{3}$, Nathan Cassidy ${ }^{4}$, Volker Ebert ${ }^{1}$, Valerio Ferracci ${ }^{4}$, Tuomas \\ Hieta $^{5}$, Daiana Leuenberger ${ }^{6}$, Nils Lüttschwager ${ }^{1}$, Nicholas Martin $^{4}$, Céline Pascale ${ }^{6}$, Carlo Tiebe ${ }^{7}$, Marsailidh M. Twigg $^{3}$, \\ Olavi Vaittinen ${ }^{8}$, Janneke van Wijk ${ }^{9}$, Klaus Wirtz ${ }^{10}$, Bernhard Niederhauser ${ }^{6}$ \\ ${ }^{1}$ Physikalisch-Technische Bundesanstalt (PTB), Bundesallee 100, 38116 Braunschweig, Germany \\ ${ }^{2}$ Dansk Fundamental Metrologi A/S (DFM), Matematiktorvet 307, DK-2800 Kgs. Lyngby, Denmark \\ ${ }^{3}$ Natural Environment Research Council, Centre for Ecology \& Hydrology (NERC), EH26 OQB, Bush Estate, Penicuik, Midlothian, United \\ Kingdom \\ ${ }^{4}$ National Physical Laboratory (NPL), TW11 OLW, Teddington, Middlesex, United Kingdom \\ ${ }^{5}$ Technical Research Centre of Finland Ltd, Centre for Metrology (VTT-MIKES), Tekniikantie 1, 02150 Espoo, Finland \\ ${ }^{6}$ Federal Institute of Metrology (METAS), Lindenweg 50, 3003 Bern-Wabern, Switzerland \\ ${ }^{7}$ BAM Federal Institute for Materials Research and Testing, Unter den Eichen 87, 12205 Berlin, Germany \\ ${ }^{8}$ University of Helsinki, Department of Chemistry, A.I. Virtasen aukio 1, P.O. Box 55, 00014, Helsinki, Finland \\ ${ }^{9}$ Dutch Metrology Institute (VSL), Thijsseweg 11, 2629 JA Delft, The Netherlands \\ ${ }^{10}$ Umweltbundesamt (UBA), Wörlitzer Platz 1, 06844 Dessau-Roßlau, Germany
}

\begin{abstract}
Résumé. Dans le cadre du Programme de Recherche Européen en Métrologie (EMRP), un projet de trois ans a débuté en juin 2014 avec l'objectif d'améliorer la compréhension et la traçabilité métrologique des mesures d'ammoniac (NH3) ambient, dans le domaine $0.5-500 \mathrm{nmol} / \mathrm{mol}$. Les trois tâches principales de ce projet sont 1) d'améliorer l'exactitude et la stabilité de mélanges de gaz de référence statiques et dynamiques, 2) de développer un étalon de transfert optique et 3) d'établir un lien entre les standards métrologiques de haute précision et les mesures de terrain. Les résultats attendus de ce projet vont améliorer l'exactitude et la fiabilité des estimations d'émissions et ainsi aider à l'évaluation de l'efficacité des mesures de réduction d'émission prescrites par les réglementations internationales. Dans cette publication nous décrivons le concept, les objectifs et les premiers résultats du projet.
\end{abstract}

\section{Introduction}

The average background concentration of ammonia in ambient air is in the range of $0.1-5 \mathrm{nmol} / \mathrm{mol}(0.1-$ $5 \mathrm{ppb}$ ), and it increases up to the order of $100 \mathrm{nmol} / \mathrm{mol}$ only in the direct vicinity of agricultural facilities or activities. Despite the low amount fractions, measuring ammonia in ambient air is a key issue in environmental science. Ammonia can have harmful effects on ecosystems and biodiversity through influencing acidity of natural waters and soils, providing excess nitrogen input to ecosystems, and on human health through influencing secondary aerosol formation. Regulations exist to control ammonia emissions in many EU member states, under the Directive 2008/1/EC of the European Parliament and of the Council concerning Integrated Pollution Prevention and Control (IPCC).

Despite the clear need set by regulations, the majority of analytical techniques which underpin or validate ammonia emission estimates lack thorough uncertainty analyses and quality assurance. Measurements are challenging due to the low concentrations of ammonia in ambient air and the relatively high concentrations of potential interfering atmospheric components, in particular water vapour. Furthermore, the highly adsorptive properties of gas-phase ammonia raise difficulties in the construction of sampling inlets for analyzers, as well as for the preparation of reference gas mixtures. Inter-comparison experiments have shown significant discrepancies between different measurement methods[1], and the agreement even between reference materials provided by European National Metrology Institutes (NMIs) is unsatisfactory[2].

MetNH3[3] (Metrology for ammonia in ambient air) is a three-year project that started in June 2014 within the framework of the European Metrology Research Programme (EMRP). The project aims to reduce the gap between requirements set by European emission regulations and the current state-of-the-art of analytical methods and reference materials. The work in the project involves six European NMIs (from Switzerland (METAS), Germany (PTB), the Netherlands (VSL), United Kingdom (NPL), Finland (VTT-MIKES) and Denmark (DFM)), two institutes designated for certain metrological tasks (the Environmental Protection Agency (UBA) and the Federal Institute for Materials Research and Testing (BAM) in Germany), as well as two research institutes (the Centre for Ecology and Hydrology (CEH) in the UK and the University of Helsinki (UH) in Finland). The project is further supported by 14 collaborators from academia and industry.

\footnotetext{
${ }^{a}$ Corresponding author: andrea.pogany@ptb.de
} 
The project is organized in three technical work packages. The first work package investigates the preparation of reference gas mixtures using different methods. The aim of this work package is to achieve traceability and reduce uncertainties in both static and dynamic reference gas mixtures. Particular emphasis is put on the minimization of adsorption losses. In the second work package optical measurement methods are studied and developed. A commercial extractive spectrometer and a self-developed open path spectrometer are examined from a metrological point of view and their applicability as traceable optical transfer standards are evaluated. Selected reference gas mixture(s) and optical transfer standard(s) from the first two work packages will be used in the third work package, which deals with dissemination to field measurement techniques. Two test facilities are being developed in this work package to serve as the infrastructure for comparison and validation experiments.

\section{Background of the research}

\subsection{Requirements by European regulations regarding ammonia emission reduction}

Together with Central and Eastern European countries, the EU, United States and Canada have negotiated the "multi-pollutant" protocol to Abate Acidification, Eutrophication and Ground-level Ozone (the so-called Gothenburg protocol, agreed in November 1999[4]) under the Convention on Long-Range Transboundary Air Pollution (cLRTAP). Annex IX of the protocol defines the measures to control ammonia emissions from agricultural sources and reduce emissions under the emission ceilings determined for each country until 2010. A revision of the Gothenburg protocol was agreed in 2012 ([5]) to continue emission reductions until 2020. Furthermore, agricultural facilities need to comply with the IPPC Best Available Techniques summarised in a reference document published by the European Commission (BREF published July 2003 by the European IPPC Bureau), the use of other abatement techniques need to be supported by evidence of effectiveness.

The Gothenburg Protocol is implemented in the EU through the National Emissions Ceiling Directive (NEC, $2001 / 81 / E C)$ to have targeted reductions of ammonia emissions. The emission ceilings in the NEC directive are equally or more ambitious than those in the Gothenburg Protocol. As an example, the sum of the $\mathrm{NH}_{3}$ emission ceilings for the 15 signing European countries is $3110 \mathrm{kt} /$ year.

Besides emission ceilings, critical levels of ammonia have also been defined for sensitive ecosystems[6]. These are based on experimental evidence and are as low as $1 \mu \mathrm{g} / \mathrm{m}^{3}(\sim 1.4 \mathrm{nmol} / \mathrm{mol}$, annual mean concentration) for ecosystems dominated by lichens and bryophytes and $3 \mu \mathrm{g} / \mathrm{m}^{3} \quad(\sim 4 \mathrm{nmol} / \mathrm{mol})$ for other ecosystems.

The European Monitoring and Evaluation Programme (EMEP[7]), directed by the United Nations
Economic Commission for Europe (UNECE) was founded to support governments and subsidiary bodies under the cLRTAP convention. A broad network of scientists and experts contribute to the collection, analysis and reporting of emission data. Further national monitoring networks with higher spatial resolution (e.g. Measuring Ammonia in Nature (MAN) Network in the Netherlands, and the National Ammonia Monitoring Network (NAN) in the UK) provide additional data to assess ambient ammonia concentrations and trends. Besides these long-term monitoring activities with lower temporal and spatial resolution, shorter measurement campaigns with higher temporal resolution are organized as well to address specific questions[1,8,9]. Countries currently adopt a variety of approaches; there is no European standard for ammonia monitoring.

\subsection{State-of-the-art of ammonia reference gas mixtures}

\subsubsection{Cylinders for the $10-100 \mu \mathrm{mol} / \mathrm{mol}$ range}

Ammonia reference gas mixtures are available commercially at amount fractions of $10 \mu \mathrm{mol} / \mathrm{mol}$ or even lower; however the uncertainties at the lowest amount fractions can be as high as $10 \%$ and the stability is guaranteed only for 6-12 months. Despite of these restrictions, gas mixtures prepared by dynamic dilution from these mixtures are routinely used to calibrate ambient ammonia monitoring instruments.

The high uncertainty of the reference gas concentration is due to the reactivity of ammonia, which causes issues with the typical approach taken for gravimetric preparation of gas mixtures (as detailed in ISO 6142 [10]). Since the largest obstacle to the development of accurate ammonia reference standards is the adsorption of ammonia onto the internal surfaces, most cylinder manufacturers offer cylinders that have undergone internal passivation treatments specific for ammonia. However; a systematic assessment and comparison of these treatments has not been published yet.

\subsubsection{Dynamic generators for amount fractions in the $\mathrm{nmol} / \mathrm{mol}$ range}

Currently available mobile generators for gas mixtures use a method based on the specific temperaturedependent permeation of the reference substance through a membrane into a flow of carrier gas, e.g. nitrogen or air, as described in the ISO 6545-10 standard[11]. This is the method of choice for the dynamic generation of gas mixtures in the amount fraction range of $\mu \mathrm{mol} / \mathrm{mol}$ to $\mathrm{nmol} / \mathrm{mol}$ with a well-defined uncertainty. Devices from several manufacturers are commercially available $[12,13,14,15]$ and used for the calibration of on-line analyzers in laboratories and the field. However, the commercial permeation devices are not specifically built for generating $\mathrm{NH}_{3}$ gas mixtures and therefore, lack some technical prerequisites. We have found none of the commercially available generators fulfilling all the 
criteria of concentration range with sufficiently low uncertainty and traceability.

\subsection{State-of-the-art of currently used ammonia monitoring methods}

\subsubsection{Non-spectroscopic analyzers}

Long-term monitoring of ammonia at remote sites is usually performed using passive or active samplers[16], which chemically bond ammonia from ambient air. The collected ammonia is subsequently analyzed in a laboratory as dissolved ammonium. Passive samplers collect ammonia by diffusion, and typically provide a time resolution of one week to three months. Active samplers are attached to a pump that ensures a continuous air flow through the sampler and, as a result of contact to a higher volume of air in a given time, allow temporal resolution down to one hour. After exposure the samplers are transported to a laboratory, where the collected ammonia is released as ammonium into a solution and subsequently analyzed by flow injection analysis, spectrophotometry or ion chromatography.

For applications requiring higher time resolution and on-line measurement, a series of wet chemical analyzers have been developed led by the Netherlands since the 1990s[17,18,19]. The most important advantages of these instruments are practically artefact-free gas handling, and the possibility of reliable and accurate calibration using liquid ammonium-solutions. Such instruments have been used for ambient ammonia monitoring in the Netherlands for decades [20], and similar instruments have been used in a number of measurement campaigns, even though their operation is quite labour intensive and consumes a high amount of chemicals.

Chemiluminescence and chemical ionization mass spectrometry (CIMS)[21] are also occasionally used techniques, especially in shorter campaigns or airborne measurements.

\subsubsection{Spectroscopic measurement methods}

Spectroscopic measurement techniques combine ease of operation and low maintenance costs with high selectivity and sensitivity and are therefore generally preferred in trace gas monitoring. Mainly driven by the rapid development of near-infrared (NIR) and midinfrared (MIR) lasers, spectroscopic measurement techniques have undergone an intensive development in the past ten years. As a result, several commercial spectrometers have become available for ambient ammonia monitoring. These spectrometers are based on measurement techniques including quantum cascade laser absorption spectroscopy[22] (QCLAS), cavity enhanced spectroscopy[23,24] (CES) and photoacoustic spectroscopy[25,26] (PAS). Commercial instruments are robust and easy to operate, and are now quite widely used for ambient ammonia measurement. However, it has been indicated that some of them may have biases caused by inadequate sampling or interference from common atmospheric components[27].
Research instruments based on QCLAS and PAS have been used in short measurement campaigns and showed good performance[1]. Even open path spectrometers (using UV[28] or MIR[29] light) are being developed to overcome sampling problems. However, many of these instruments are still in the research phase, i.e. they are very complex and need to be operated by experts.

\section{Research challenges}

\subsection{Challenges in preparing reference gas mixtures}

\subsubsection{Stability and accuracy of gas mixtures in cylinders}

Due to the highly adsorptive nature and reactivity of ammonia, adsorption to cylinder walls and chemical reaction with impurities in the matrix gas are the main difficulties encountered in the gravimetric preparation of ammonia primary reference mixtures. The efforts of the gas metrology community to tackle these issues are aptly summarised in the report of the international key comparison CCQM-K46 [2]. In this comparison exercise, mixtures with nominal amount fraction over the range of $30-50 \mu \mathrm{mol} / \mathrm{mol}$ were distributed to the participating NMIs, who in turn certified these mixtures using their own in-house reference standards and methods. The results are summarised in Figure 1. Whilst a certain level of agreement between the different methods used to certify the mixtures can be observed, the overall discrepancies are also obvious.

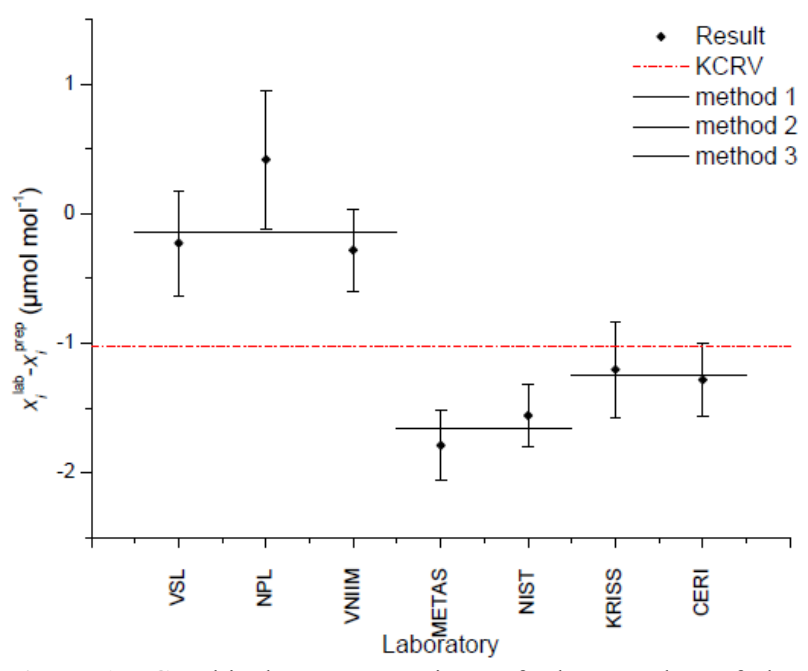

Figure 1: Graphical representation of the results of key comparison CCQM-K46 (ammonia in nitrogen). The red dotdashed line indicates the reference value for the comparison (KCRV). Figure taken from [2].

The disagreement between the results was attributed to a number of reasons, including the different cylinder passivation techniques used by the participating NMIs to produce their own reference mixtures, and the different analytical techniques used to perform the analysis. As an 
example, it was discussed that comparing the dynamic preparation by permeation and subsequent dilution where a continuous gas flow is maintained over several hours with measurements on gas cylinders done over much shorter periods might lead to discrepancies. The lack of consensus amongst NMIs may result in poor instrument calibration and would affect the comparability of national measurement networks. We note that in order to improve these results a further key comparison (CCQM K-117) is planned and will be organized by VSL and NIST in 20152016.

\subsubsection{Concentration and uncertainty limits in dynamic generators}

The generation of $\mathrm{NH}_{3}$ amount fractions in the range of $0.5-500 \mathrm{nmol} / \mathrm{mol}$ by permeation with low uncertainties requires extreme stability and precision in the control parameters, such as mass $(m)$, temperature $(T)$ and gas flow rate $(Q)$. Moreover, an additional dilution step is required to achieve $\mathrm{NH}_{3}$ amount fractions of $0.5 \mathrm{nmol} / \mathrm{mol}$ placing considerable demands on the pressure control of a device. Commercially available gas generators produce gas mixtures with uncertainties not well-defined and considerably higher than $3 \%$.

\subsection{Challenges of the analytical methods}

\subsubsection{Gas sampling}

Adsorption studies [30,31,32], of ammonia on glass, metal and polymer surfaces have shown that surface material has a drastic effect on adsorption and thereby on the ammonia amount fraction in the gas sample. For inlet lines adsorption is a strong function of temperature, relative humidity, residence time and pressure. Adding a commercial coating (e.g. SilcoNert 2000, Silcotek Corporation) on stainless steel reduces its adsorption by up to a factor of 25[30] (see Figure 2), and polymer surfaces adsorb even less ammonia than coated metal surfaces. As inlet lines of analyzers the most preferred materials are polymers (e.g. PTFE or PVDF), whereas gas cells, valves and flow control units - where more mechanical stability is required - are often built using coated stainless steel or pyrex. Studies into using an "active passivation method" by entraining functionalized perfluoroalkane vapour into the inlet sampling stream are also currently underway to reduce the absorption effects in spectroscopic analyzers; these are, however, still in the research phase[33].

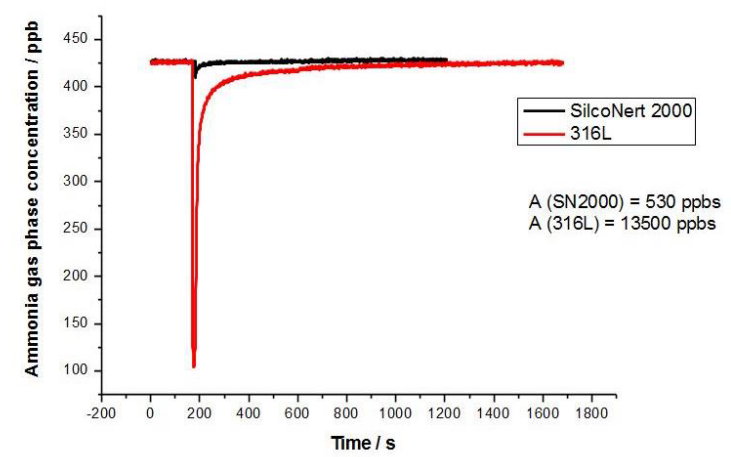

Figure 2: Ammonia gas phase concentration as a function of time in an experiment where a clean metal tube is abruptly exposed to ammonia with a concentration of $425 \mathrm{nmol} / \mathrm{mol}$. The sudden drop in the signal is due to the adsorption and gas exchange occurring in the tube.

Heating the sampling lines or the measurement cell is a quite common method to further decrease adsorption losses and prevent condensation. However, there are concerns that elevated temperature leads to a partitioning of aerosol, such as $\mathrm{NH}_{4} \mathrm{NO}_{3}$, into $\mathrm{NH}_{3}$ and $\mathrm{HNO}_{3}$ in the gas phase, leading to a positive bias in the measurements[1]. The use of filters and impactors to remove aerosols from the air stream prior to entering the heated sampling line is a widespread method to reduce this positive bias, as well as to prevent contamination of the measurement cell. In this case it is important that filters are changed regularly to prevent reaction of gas phase ammonia with the aerosol phase captured on the filter, or volatilization of the captured aerosols.

\subsubsection{Spectroscopic challenges}

Careful spectral line selection is a crucial point in the development of spectroscopic analyzers, due to severe spectral interference from common atmospheric components, like water vapour $\left(\mathrm{H}_{2} \mathrm{O}\right)$, carbon dioxide $\left(\mathrm{CO}_{2}\right)$, ozone $\left(\mathrm{O}_{3}\right)$ and methane $\left(\mathrm{CH}_{4}\right)$. Figure 3 gives an overview of the infrared spectrum of ammonia and common atmospheric components.

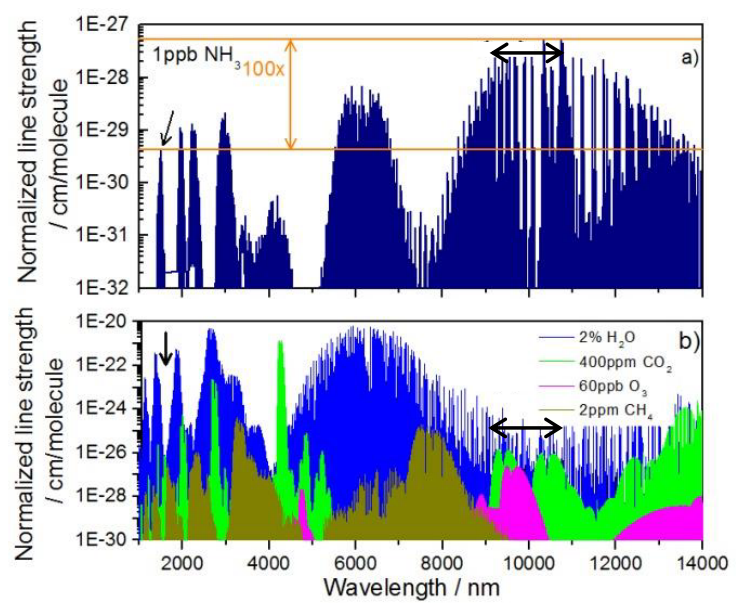

Figure 3: Infrared spectrum of $\mathrm{NH}_{3}$ (a) and common atmospheric interferents (b). The commonly used wavelength ranges are marked with arrows. Normalized line strength refers to the line strengths multiplied by typical ambient concentrations (see legend). 
Two wavelength ranges (indicated by arrows in Figure 3) are frequently used for ambient ammonia analysis by spectroscopy, both having their advantages and disadvantages. The wavelength range around $1.5 \mu \mathrm{m}$ $\left(6500-6600 \mathrm{~cm}^{-1}\right)$ has been accessible for decades using robust diode lasers produced for the telecommunication industry. However, the rather small line strength of the ammonia lines in this range $\left(<2 \times 10^{-21} \mathrm{~cm} /\right.$ molecule $)$ requires the use of extremely sensitive detection techniques (CES or PAS). On the other hand, measurements using the strongest, MIR ammonia absorption lines around $9-11 \mu \mathrm{m}\left(900-1100 \mathrm{~cm}^{-1}\right)$ was hindered for a long time by the limited availability of light sources. Earlier, only line tuneable carbon dioxide lasers and lead salt diodes requiring cryogenic cooling were available in this wavelength range. However; the rapid development of room temperature quantum cascade lasers makes measurements in this wavelength range more feasible.

To overcome the problem of limited accuracy and availability of calibration standards (as described in Section 2.2.1 and 2.2.2), many spectrometers aim to achieve calibration-free, absolute measurements based on the Beer-Lambert law[29,34]. This approach relies on the accurate knowledge of the spectral line parameters (most importantly the line strengths and pressure broadening coefficients) of the probed transitions. The quality of available spectral line data is however not satisfactory. As an example, until 2012 the HITRAN database[35] (the most frequently used spectroscopic database in atmospheric science) did not contain any data of ammonia for wavelengths below $2 \mu \mathrm{m}$, and even in the 2012 edition of the database, uncertainties of the ammonia lines are typically as high as $5-20 \%$. More detailed studies of individual, application-specifically selected absorption lines (e.g. [36]) provide only slightly lower uncertainties.

\section{Ammonia and metrology: current developments and aims}

\subsection{Gas mixtures}

\subsubsection{Cylinders for the $10-100 \mu \mathrm{mol} / \mathrm{mol}$ range}

The MetNH3 project aims to overcome the current limitations in the development of accurate and traceable ammonia standards. As described in Section 2.2 and 3.1, gravimetric preparation of ammonia standards is often complicated by the adsorption of ammonia molecules onto the gas cylinder internal surfaces. Cylinders can undergo a number of passivation treatments in order to minimise adsorption onto walls; however these treatments are component-specific and must be assessed by preparing mixtures gravimetrically and measuring the ammonia in the output gas. The first step in the current study was to assess the performance of a range of commercially available cylinder passivation techniques, to determine which one is best suited for the development of static ammonia primary reference mixtures. Several mixtures were prepared in different type of cylinders, the stability of which will be examined in the course of the project.

\subsubsection{Dynamic generators for $\mathrm{nmol} / \mathrm{mol}$ amount fractions}

A further aim of MetNH3 is to dynamically generate traceable certified reference material (CRM, according to ISO/IEC Guide 99:2007) in typical ambient $\mathrm{NH}_{3}$ amount fractions $(0.5-500 \mathrm{nmol} / \mathrm{mol})$ with a relative uncertainty below $3 \%$. The generator is based on a permeation oven, however, $\mathrm{NH}_{3}$ amount fractions down to $0.5 \mathrm{nmol} / \mathrm{mol}$ can only be generated in a mobile device where the base mixture prepared by permeation is diluted in at least two more steps.

There are several criteria that need to be fulfilled for achieving this goal. First of all, all relevant parameters of the generator, such as mass $(m)$, time $(t)$, temperature $(T)$, gas flow $(Q)$ and permeation rate $(\mathrm{Pr})$ of the permeation device have to be traceable to NMI standards. This requires the accessibility of all relevant parts of the mobile device for calibration purposes (e.g. temperature sensor in the permeation oven, mass flow controllers and the permeation device).

In order to achieve the ambitious aim of uncertainty $<3 \%$, the individual extended $(k=2)$ uncertainties of the following parameters must not be exceeded: permeation rate $(<1.5 \%)$, dilution $(<0.6 \%)$ and temperature $(<2 \%)$. Considerable uncertainty, particularly at lower amount fractions is added by the residual $\mathrm{NH}_{3}$ in the used matrix gas (typically $0.05 \pm 0.05 \mathrm{nmol} / \mathrm{mol}$ ).

As mentioned before, $\mathrm{NH}_{3}$ readily adsorbs and desorbs on material surfaces such as stainless steel. A new coating material has been successfully tested [16] and in order to minimize said effects as well as stabilisation time, all surfaces in contact with the $\mathrm{NH}_{3}$ CRM ought to be coated. This will considerably reduce adsorption-desorption effects and result in a faster equilibration time of stable $\mathrm{NH}_{3}$ CRM.

Two mobile gas generators for the production of CRM of $\mathrm{NH}_{3}$ are being developed in the framework of MetNH3. Based on the achievements of the project, a good practice guide will be compiled on the accurate preparation of ammonia gas standards for environmental measurements.

\subsection{Spectroscopic measurement methods}

\subsubsection{Absolute, extractive spectroscopic techniques}

The MetNH3 project aims to develop optical transfer standard(s) for ambient ammonia amount fraction measurements. Our recent studies show that absolute spectroscopic measurement techniques (tunable diode laser absorption spectroscopy (TDLAS) or cavity ringdown spectroscopy (CRDS)) provide the potential to achieve traceability in spectroscopic amount fraction measurements without the need for calibration using gas standards $[37,38]$. Based on absolute spectroscopic 
techniques an optical transfer standard can be developed, which is independent from gas standards.

Determination of the $\mathrm{NH}_{3}$ amount fraction $\left(x_{\mathrm{NH} 3}\right)$ is based on the Beer-Lambert law according to the following equation:

$$
x_{\mathrm{NH}_{3}}=\frac{T \cdot A_{\text {line }} \cdot k_{B}}{S_{T} \cdot L \cdot p \cdot r_{\text {iso }}}=\frac{T \cdot \alpha_{\text {int }} \cdot k_{B}}{S_{T} \cdot p \cdot r_{\text {iso }}} .
$$

Traceability of the input parameters optical path length $(L)$, pressure $(p)$ and temperature $(T)$ of the gas sample has already been achieved[37]. $k_{\mathrm{B}}$ is the Boltzmannconstant, which is known with very high accuracy. The isotopic ratio (represented by the correction factor $r_{\text {iso }}$ ) of natural gas samples can be estimated with high accuracy based on natural abundances[39]. Usually the line strength of the probed transition $\left(S_{T}\right)$ and the integrated absorbance or absorption coefficient ( $A_{\text {line }}$ in TDLAS and $\alpha_{\text {int }}=A_{\text {line }} / L$ in CRDS) are the two most challenging parameters, which are addressed in Section 4.2.3.

In extractive analyzers, adsorption processes in the sampling system or in the measurement cell might affect response time, accuracy and uncertainty, which effects have to be examined in more detail. As described in Section 3.2.2, several studies deal with the effect of different materials. However, the influence of temperature or humidity (competitive adsorption between ammonia and water) on the adsorption processes is less clear. Certain studies indicate lower adsorption on polymer surfaces at higher temperatures[31], which would agree with the thermodynamic properties of ammonia. The effect of water vapour on ammonia adsorption is unclear. Vaittinen et al. [30] observed that increased water vapour concentration causes a remarkable decrease in adsorption losses of ammonia, while experiments performed by Ellis et al. [40] show the opposite. The ammonia-water-surface interplay will be studied experimentally in detail in the MetNH3 project to resolve this conflict.

\subsubsection{Open path spectrometer}

Part of the MetNH3 project is investigating whether sampling-free laser spectroscopic measurement techniques are suitable to be used as an optical transfer standard in ambient ammonia measurements. An open or a sampling-free spectrometer does not suffer from adsorption problems that may severely limit the use of conventional extractive instruments as discussed in the previous section. So far the most advanced work towards compact and sensitive sampling-free ammonia sensors have been published by Volten et. al. in 2012 [28] and by Miller et. al. in 2014 [29]. However, we note that both spectrometers need calibration, and no published results of an absolute sampling-free ammonia analyzer exist to our knowledge.

The spectrometer developed in MetNH3 is based on a QCL operating close to $1103.5 \mathrm{~cm}^{-1}$, which enables the use of a strong and fairly isolated ammonia feature. The feature consists of multiple unresolved transitions, which will be characterized in MetNH3 project. A preliminary design on the sampling-free spectrometer is shown in Fig. 4.

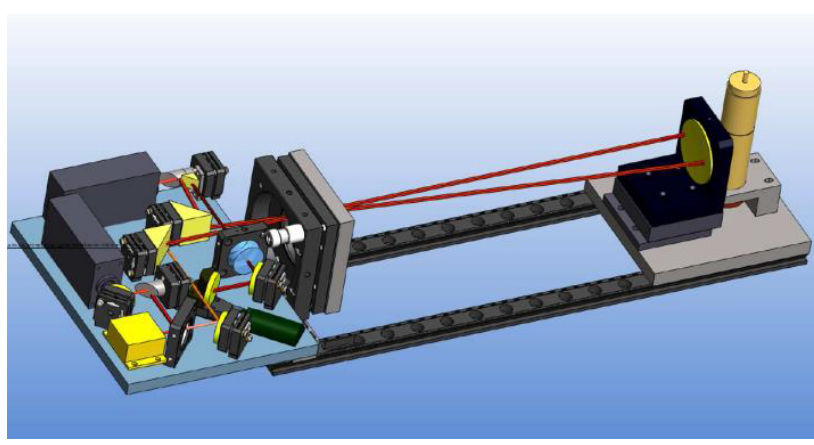

Figure 4: Preliminary design of a sampling-free spectrometer showing only input and output beams inside the multipass cell

The spectrometer consists of a Herriot type multipass cell. To minimize optical fringes, the cell design will be of non-standard type and mirror dithering will be tested to further reduce the problems caused by fringes. A moving mirror is used to validate the tuning rate of the QCL using an etalon, which is required for absolute amount fraction measurements. The target sensitivity of the instrument is below $1 \mathrm{nmol} / \mathrm{mol}$ using $60 \mathrm{~s}$ measurement time.

\subsubsection{Spectral line data and line modelling}

Both extractive and open path spectrometers developed in the project rely on accurate spectral line parameters. Previously, a robust and flexible method has been developed to measure line strengths of $\mathrm{CO}_{2}$ and $\mathrm{H}_{2} \mathrm{O}$ by TDLAS with expanded uncertainties $(k=2)$ in the 1-3 \% range[41]. The application of a similar method for ammonia is foreseen later in the project. Similarly, pressure broadening coefficients have been measured[42] using a method, which can also be applied for ammonia.

Due to the number of interfering molecules, fitting of the measured absorption spectra, and thereby determining $A_{\text {line }}$ or $\alpha_{\text {int }}$, is not straightforward. A multiline fitting algorithm is being developed for the spectral ranges used in the MetNH3 project, taking into account the relevant absorption lines of $\mathrm{NH}_{3}, \mathrm{H}_{2} \mathrm{O}$ and $\mathrm{CO}_{2}$ [43]. Figure 5 shows an example spectrum with the fitted lines and residuals, measured with an extractive CRDS spectrometer. A reasonably good fit has been achieved by fitting three $\mathrm{NH}_{3}$ and six $\mathrm{H}_{2} \mathrm{O}$ lines, but further improvements are anticipated.

Uncertainty of the integrated absorption coefficient is dominated by uncertainty of the measured ring-down times, and is in the $0.5-9 \%$ range, depending on the $\mathrm{NH}_{3}$ and $\mathrm{H}_{2} \mathrm{O}$ amount fractions. This component is, besides the $\sim 10 \%$ uncertainty in the line strength (currently used literature values) the most important contributor to the uncertainty of the amount fraction result. Considerable decrease in these uncertainties is foreseen later on in the project. 


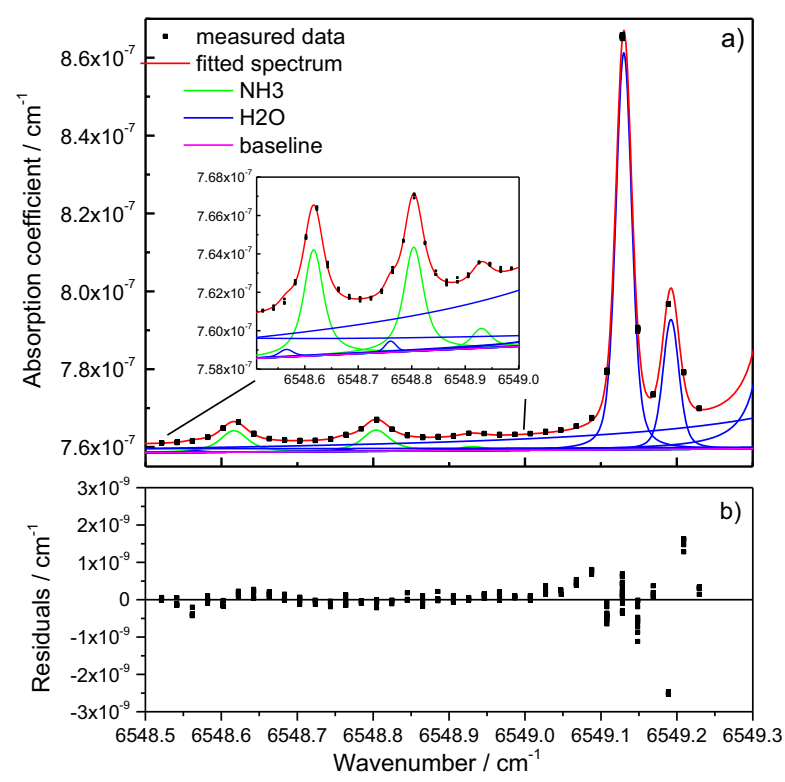

Figure 5: Measured data points with the fitted spectrum (a) and residuals (b) in $100 \mathrm{nmol} / \mathrm{mol} \mathrm{NH}_{3}$ and $2 \% \mathrm{H}_{2} \mathrm{O}$ in $\mathrm{N}_{2}$.

As can be seen in Figure 5, the $\mathrm{NH}_{3}$ absorption lines strongly overlap with absorption lines of $\mathrm{H}_{2} \mathrm{O}$. Spectral interference is expected due to two reasons: a) direct overlap of absorption lines and $b$ ) change in the width of the $\mathrm{NH}_{3}$ absorption lines due to significantly changing $\mathrm{H}_{2} \mathrm{O}$ concentration in the matrix gas. This crosssensitivity has been investigated and found to be not negligible. Figure 6 shows two calibration lines of a CRDS instrument: one in dry and one in humidified gas samples (the samples were prepared in the CATFAC facility; see Section 4.3. for more details). The slope of the two calibration lines differ by $\sim 4 \%$, which is higher than the expanded measurement uncertainty of up to $\pm 2.3 \%$ and clearly indicates cross-sensitivity to $\mathrm{H}_{2} \mathrm{O}$. An improved data evaluation algorithm has been developed in cooperation with the analyzer manufacturer (Picarro Inc.[23]) within the MetNH3 project to ensure accurate $\mathrm{NH}_{3}$ amount fraction readings in humid gas samples.

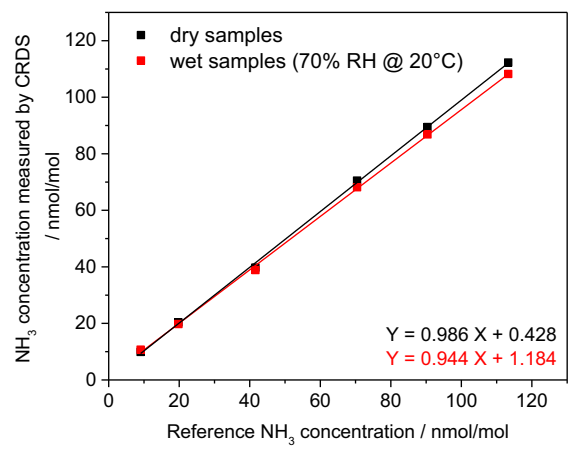

Figure 6: Calibration lines of a CRDS instrument (lack of fit plot) for dry and humidified ammonia atmosphere tests.

\subsection{Comparison and validation experiments}

NPL has developed a Controlled Atmosphere Test Facility (CATFAC), for carrying out exposure tests with multiple types of ammonia samplers, as a validation of measurement techniques that can be applied in the field.
A photograph of the CATFAC, without its insulation, is shown in Figure 7. The facility is an aerodynamic wind tunnel constructed of borosilicate glass, which is designed to incorporate individual adjustment of parameters such as concentration, relative humidity, temperature and air speed. The specified conditions can easily be maintained over time intervals of a few hours to several weeks.

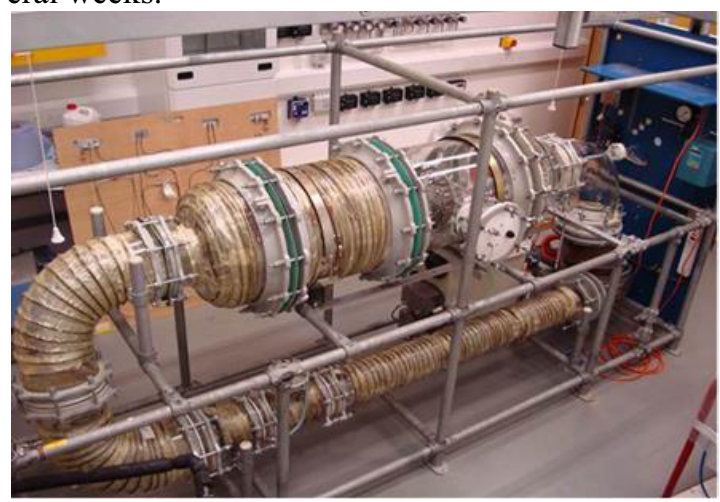

Figure 7: Photograph of the Controlled Atmosphere Test Facility (CATFAC)

Test atmospheres are generated by dynamically blending (through calibrated mass flow controllers) stable ammonia Primary Standard Gas Mixtures (PSM), prepared at NPL, with purified air. The test gas is continuously re-circulated and replenished in the facility by a freshly generated mixture of the same composition. The total replenishment flow rate is up to $30 \mathrm{~L} \mathrm{~min}^{-1}$ thereby allowing diffusive samplers, pumped denuders and on-line optical analysers to be deployed simultaneously. The first test measurement using a CRDS spectrometer [23] and denuders have already been performed and showed good results.

UBA operates a proficiency test facility to characterise the performance of ambient air monitors used in national networks to measure air quality data. The facility consists of a dynamic dilution system connected to a glass line with multiple sampling ports for the individual instruments (a photograph shown in Figure 8). Complex gas mixtures can be generated by the dilution of up to 14 pressurized gas cylinders with time-programmed step changes in the concentration of the individual mixtures by using mass flow controllers interfaced to an industrial computer. In addition an ambient air line can be used to provide air from outside the building to the connected instruments

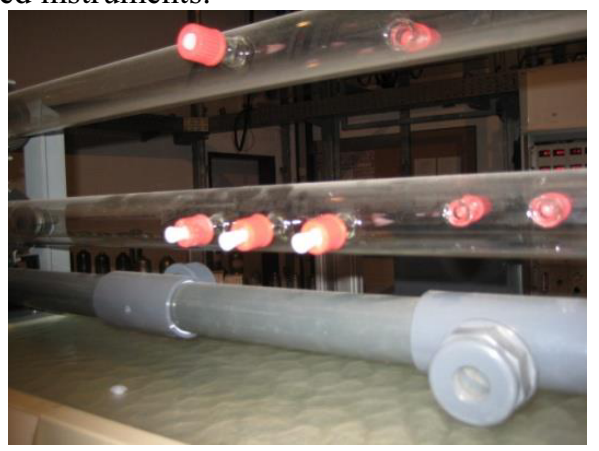

Figure 8: Photograph of the two glass sampling lines at the proficiency test facility at UBA. The upper glass line connects to ambient air from outside the building 
The proficiency test facility will be used to test the newly developed instruments with respect to potential interference gases in order to select the most suitable transfer standard for ambient measurements in the field. The system has been characterised for ammonia test gases under dry and humid conditions. The installation operates under ambient temperature and pressure. Step changes in concentration will be applied to the interfering compounds. Ammonia test gases show a long stabilization time up to 6 hours due to the strong adsorption effects on the glass walls, especially under humid conditions.

Historically there have been a number of field intercomparisons of both research and commercial $\mathrm{NH}_{3}$ instrumentation[1,8,44]. These studies have demonstrated that under field conditions additional complications arise which are not observed under controlled conditions in the laboratory. The use of unheated inlets has been observed to lead to an underestimation in $\mathrm{NH}_{3}$ amount fractions when condensation occurs, however in heated inlet lines and with particulate filters, conditions can lead to an overestimation [1]. Atmospheric variables such as temperature, pressure, relative humidity and aerosol loading are also known to result in variable results. For example, traditional wet chemistry methods using flow injection analysis suffer from calibration errors due to fluctuating temperature. All the above complicating parameters can lead to reduced temporal responses in instruments.

The most recent inter-comparison was organized in 2008 and as highlighted in the present article there has been a significant effort in developing commercial methodologies since then. In order to test the gas standards and the analytical methodologies developed in MetNH3 an international field inter-comparison will be coordinated in 2016. Both passive and active off-line samplers will be put in parallel with on-line instrumentation. It is understood that for field applications the method adopted is directed by the science hypothesis being tested; however understanding the trade-offs in temporal and spatial resolution, uncertainty of measurements and economic costs are important considerations too. Recommendations for best practice and instrument capabilities will be discussed and developed from all of the MetNH3 studies.

\section{Conclusions and outlook}

Previous studies revealed significant discrepancies between reference gas mixtures of $\mathrm{NH}_{3}$ in the $\mu \mathrm{mol} / \mathrm{mol}$ amount fraction range, as well as between amount fractions measured by different analytical techniques. One major reason for this is the highly adsorptive and reactive nature of ammonia, which influences both reference materials and analytical techniques. The MetNH3 project aims to improve the situation through developments in three major fields: 1) development of certified reference materials in cylinders and traceable dynamic gas mixture generators, 2) development and characterization of sampling-free and extractive spectroscopic instruments aiming to construct a traceable optical transfer standard and 3) providing infrastructure for laboratory and field inter-comparison measurements to establish the link between high-accuracy metrological standards and field measurement methods.

First results of the project have been described in this article. Different cylinder treatment techniques are being examined to decrease adsorption losses in static gas mixtures in the $10-100 \mu \mathrm{mol} / \mathrm{mol}$ amount fraction range and achieve an uncertainty better than $1 \%$. Two mobile dynamic generators are being developed to achieve better than $3 \%$ relative uncertainty in ammonia gas mixtures in the $0.5-500 \mathrm{nmol} / \mathrm{mol}$ amount fraction range. An extractive cavity ring-down spectrometer is characterized from a metrological point of view and its applicability as an optical transfer standard is being evaluated. A QCLAS spectrometer based on an open multi-pass cell is constructed, which will provide sampling-free, absolute measurement of ambient ammonia amount fractions. Two test facilities are being developed to provide an infrastructure for inter-comparison of the developed standards, as well as commonly used analyzers and samplers.

\section{References}

1. Bobrutzki K Von, Braban CF, Famulari D, et al. (2010) Field inter-comparison of eleven atmospheric ammonia measurement techniques. Atmos Meas Tech 3:91-112.

2. Veen AMH Van Der, Nieuwenkamp G, Wessel RM, et al. (2010) International Comparison CCQM K46 - Ammonia in Nitrogen. Metrologia. doi:10.1088/0026-1394/47/1A/08023

3. MetNH3 project homepage.

http://www.metnh3.eu/.

4. Protocol to the 1979 Convention on Long-range Transboundary Air Pollution to Abate Acidification, Eutrophication and Ground-Level Ozone. 1-94.

5. ECE/EB.AIR/111/Add.1, Decision 2012/12 Guidance for adjustments under the Gothenburg Protocol to emission reduction commitments or to inventories for the purposes of comparing total national emissions with them. 90-91.

6. Air Pollution Information System. http://www.apis.ac.uk/.

7. European Monitoring and Evaluation Programme (EMEP). http://www.emep.int/.

8. Norman M, Spirig C, Wolff V, et al. (2008) Intercomparison of ammonia measurement techniques at an intensively managed grassland site (Oensingen, Switzerland). Atmos Chem Phys Discuss 8:19791-19818.

9. Sutton MA, Nemitz E, Theobald MR, et al. (2009) Dynamics of ammonia exchange with cut grassland : strategy and implementation of the GRAMINAE Integrated Experiment. Biogeosciences 6:309-331.

10. ISO 6142:2006 Gas analysis. Preparation of calibration gas mixtures. Gravimetric method. 
11. ISO 6145-10:2012-06, Gas analysis - Preparation of calibration gas mixtures using dynamic volumetric methods - Part 10: Permeation method.

12. LNI Schmidlin SA. www.Inischmidlin.ch.

13. Owlstone Ltd. www.owlsotnenanotech.com.

14. VICI AG International. www.vici.com.

15. Kin-Tek Laboratories Inc. www.kin-tek.com.

16. Horváth L, Sutton M a. (1998) Long-term record of ammonia and ammonium concentrations at $\mathrm{K}$ puszta, Hungary. Atmos Environ 32:339-344.

17. Wyers GP, Otjes RP, Slanina J (1993) A continuous-flow denuder for the measurement of ambient concetrations and surface-exchange fluxes of ammonia. Atmos Environ 27A:20852090.

18. Erisman JW, Otjes R, Hensen A, et al. (2001) Instrument development and application in studies and monitoring of ambient ammonia. Atmos Environ 35:1913-1922.

19. Rumsey IC, Cowen K a., Walker JT, et al. (2014) An assessment of the performance of the Monitor for AeRosols and GAses in ambient air (MARGA): A semi-continuous method for soluble compounds. Atmos Chem Phys 14:56395658.

20. Buijsman E, Aben JMM, Van Elzakker BG, Mennen MG (1998) An automatic atmospheric ammonia network in The Netherlands: Set-up and results. Atmos Environ 32:317-324.

21. Norman M, Hansel A, Wisthaler A (2007) $\mathrm{O}_{2}{ }^{+}$as reagent ion in the PTR-MS instrument: Detection of gas-phase ammonia. Int J Mass Spectrom 265:382-387.

22. Aerodyne Research Inc. http://www.aerodyne.com/.

23. Picarro Inc. www.picarro.com.

24. LosGatos Research Inc. http://www.lgrinc.com/.

25. LSE Monitors. www.lsemonitors.nl.

26. EcoPhysics GmBH. http://www.ecophysics.com/.

27. Nara H, Tanimoto H, Tohjima Y, et al. (2012) Effect of air composition $\left(\mathrm{N}_{2}, \mathrm{O}_{2}, \mathrm{Ar}\right.$, and $\left.\mathrm{H}_{2} \mathrm{O}\right)$ on $\mathrm{CO}_{2}$ and $\mathrm{CH}_{4}$ measurement by wavelengthscanned cavity ring-down spectroscopy: Calibration and measurement strategy. Atmos Meas Tech 5:2689-2701.

28. Volten H, Bergwerff JB, Haaima M, et al. (2012) Two instruments based on differential optical absorption spectroscopy (DOAS) to measure accurate ammonia concentrations in the atmosphere. Atmos Meas Tech 5:413-427.

29. Miller DJ, Sun K, Tao L, et al. (2014) Open-path, quantum cascade-laser-based sensor for highresolution atmospheric ammonia measurements. Atmos Meas Tech 7:81-93.

30. Vaittinen O, Metsälä M, Persijn S, et al. (2013) Adsorption of ammonia on treated stainless steel and polymer surfaces. Appl Phys B 115:185-196.

31. Mukhtar S, Rose AJ, Capareda SC, et al. (2003) Assessment of ammonia adsorption onto teflon and LDPE tubing. Agric Eng Int CIGR J V:1-13.
32. Henningsen J, Melander N (1997) Sensitive measurement of adsorption dynamics with nonresonant gas phase photoacoustics. Appl Opt 36:7037-7045.

33. Roscioli J, Herndon S, Zahniser M, et al. (2015) Recent Advances in Detection of Ammonia and Nitric Acid on Short Timescales Suitable for Eddy Covariance Flux Measurements. Geophys Res Abstr 17:EGU015-7332.

34. Owen K, Farooq A (2014) A calibration-free ammonia breath sensor using a quantum cascade laser with WMS 2f/1f. Appl Phys B 116:371383.

35. Rothman LS, Gordon IE, Babikov Y, et al. (2013) The HITRAN2012 molecular spectroscopic database. J Quant Spectrosc Radiat Transf 130:4-50.

36. Owen K, Es-sebbar ET, Farooq A (2013) Measurements of $\mathrm{NH}_{3}$ linestrengths and collisional broadening coefficients in $\mathrm{N}_{2}, \mathrm{O}_{2}$, $\mathrm{CO}_{2}$, and $\mathrm{H}_{2} \mathrm{O}$ near $1103.46 \mathrm{~cm}^{-1}$. J Quant Spectrosc Radiat Transf 121:56-68.

37. Pogány A, Wagner S, Werhahn O, Ebert V (2015) Development and Metrological Characterization of a Tunable Diode Laser Absorption Spectroscopy (TDLAS) Spectrometer for Simultaneous Absolute Measurement of Carbon Dioxide and Water Vapor. Appl Spectrosc 69:257-268.

38. Buchholz B, Böse N, Ebert V (2014) Absolute validation of a diode laser hygrometer via intercomparison with the German national primary water vapor standard. Appl Phys B 116:883-899.

39. Berglund M, Wieser ME (2011) Isotopic compositions of the elements 2009 (IUPAC Technical Report). Pure Appl Chem 83:397-410.

40. Ellis R a., Murphy JG, Pattey E, et al. (2010) Characterizing a Quantum Cascade Tunable Infrared Laser Differential Absorption Spectrometer (QC-TILDAS) for measurements of atmospheric ammonia. Atmos Meas Tech 3:397406.

41. Pogány A, Ott O, Werhahn O, Ebert V (2013) Towards traceability in $\mathrm{CO}_{2}$ line strength measurements by TDLAS at $2.7 \mu \mathrm{m}$. J Quant Spectrosc Radiat Transf 130:147-157.

42. Ortwein P, Woiwode W, Wagner S, et al. (2009) Laser-based measurements of line strength, selfand pressure-broadening coefficients of the $\mathrm{H}^{35} \mathrm{Cl}$ $\mathrm{R}(3)$ absorption line in the first overtone region for pressures up to $1 \mathrm{MPa}$. Appl Phys B 100:341347.

43. Nielsen L (2002) Evaluation of measurements by the method of least squares. In: Levesley J, Andreson IJ, Mason JC (eds) Algorithms Approx. IV. University of Huddersfield, pp 170-186

44. Whitehead JD, Twigg M, Famulari D, et al. (2008) Evaluation of Laser Absorption Spectroscopic Techniques for Eddy Covariance Flux Measurements of Ammonia. Environ Sci Technol 42:2041-2046. 